\title{
Front Matter: Volume 7072
}

, "Front Matter: Volume 7072," Proc. SPIE 7072, Optics and Photonics for Information Processing II, 707201 (26 September 2008); doi:

$10.1117 / 12.812755$

SPIE Event: Optical Engineering + Applications, 2008, San Diego, California, United SPIE. States 


\section{PROCEEDINGS OF SPIE}

\section{Optics and Photonics for Information Processing II}

Abdul Ahad Sami Awwal

Khan M. Iftekharuddin

Bahram Javidi

Editors

13-14 August 2008

San Diego, California, USA

Sponsored and Published by

SPIE

Volume 7072 
The papers included in this volume were part of the technical conference cited on the cover and title page. Papers were selected and subject to review by the editors and conference program committee. Some conference presentations may not be available for publication. The papers published in these proceedings reflect the work and thoughts of the authors and are published herein as submitted. The publisher is not responsible for the validity of the information or for any outcomes resulting from reliance thereon.

Please use the following format to cite material from this book:

Author(s), "Title of Paper," in Optics and Photonics for Information Processing II, edited by Abdul Ahad Sami Awwal, Khan M. Iftekharuddin, Bahram Javidi, Proceedings of SPIE Vol. 7072 (SPIE, Bellingham, WA, 2008) Article CID Number.

ISSN 0277-786X

ISBN 9780819472922

Published by

SPIE

P.O. Box 10, Bellingham, Washington 98227-0010 USA

Telephone +1 3606763290 (Pacific Time) · Fax +1 3606471445

SPIE.org

Copyright (c) 2008, Society of Photo-Optical Instrumentation Engineers

Copying of material in this book for internal or personal use, or for the internal or personal use of specific clients, beyond the fair use provisions granted by the U.S. Copyright Law is authorized by SPIE subject to payment of copying fees. The Transactional Reporting Service base fee for this volume is $\$ 18.00$ per article (or portion thereof), which should be paid directly to the Copyright Clearance Center (CCC), 222 Rosewood Drive, Danvers, MA 01923. Payment may also be made electronically through CCC Online at copyright.com. Other copying for republication, resale, advertising or promotion, or any form of systematic or multiple reproduction of any material in this book is prohibited except with permission in writing from the publisher. The CCC fee code is 0277-786X/08/\$18.00.

Printed in the United States of America.

Publication of record for individual papers is online in the SPIE Digital Library.

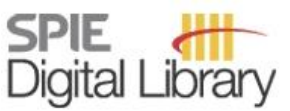

SPIEDigitalLibrary.org

Paper Numbering: Proceedings of SPIE follow an e-First publication model, with papers published first online and then in print and on CD-ROM. Papers are published as they are submitted and meet publication criteria. A unique, consistent, permanent citation identifier (CID) number is assigned to each article at the time of the first publication. Utilization of CIDs allows articles to be fully citable as soon they are published online, and connects the same identifier to all online, print, and electronic versions of the publication. SPIE uses a six-digit CID article numbering system in which:

- The first four digits correspond to the SPIE volume number.

- The last two digits indicate publication order within the volume using a Base 36 numbering system employing both numerals and letters. These two-number sets start with $00,01,02,03,04,05$, $06,07,08,09,0 \mathrm{~A}, 0 \mathrm{~B} \ldots \mathrm{OZ}$, followed by 10-1Z, 20-2Z, etc.

The CID number appears on each page of the manuscript. The complete citation is used on the first page, and an abbreviated version on subsequent pages. Numbers in the index correspond to the last two digits of the six-digit CID number. 


\section{Contents}

ix Conference Committee

\section{SESSION 1 SWITCHING}

707202 All-optical swapping of spectral amplitude code labels for packet-switched networks (Invited Paper) [7072-01]

L. R. Chen, McGill Univ. (Canada)

707203 Liquid crystal-based dynamic channel blocker/equalizer for optical networks [7072-02] S. Wang, Univ. of North Texas (United States); C.-H. Cheng, Miami Univ. (United States); Y. LU, Nanjing Univ. (China); C. Wong, RadiSys Co. (United States)

707204 Applying the triple correlation functions to characterizing high-frequency repetition trains of picosecond optical pulses [7072-03]

A. L. Muñoz Zurita, A. S. Shcherbakov, E. Tepichin Rodriguez, National Institute for Astrophysics, Optics and Electronics (Mexico); S. A. Nemov, St.-Petersburg State Polytechnical Univ. (Russia); J. Campos Acosta, CSIC—Institute for Applied Physics (Spain)

707206 Application of the effect polarization self-modulation for processing an output signal onefiber few-mode interferometers [7072-05]

V. A. Kolchinskiy, Far Eastern State Technical Univ. (Russia)

707207 A two-pass Sagnac loop for high-contrast ultrafast switching at 1053nm [7072-38] A. Jolly, L. Lago, S. Jonathas, J. Luce, H. Coic, J. F. Gleyze, CEA, Centre d'Etudes Scientifiques et Techniques d'Aquitaine (France); P. Le Boudec, IDIL Fibres Optiques (France); N. Traynor, PERFOS Lannion (France)

\section{SESSION 2 HOLOGRAPHY/MODELING}

707208 Removing the twin image in digital holography by segmented filtering of in-focus twin image [7072-07]

C. McElhinney, B. M. Hennelly, L. Ahrenberg, National Univ. of Ireland, Maynooth (Ireland); T. J. Naughton, National Univ. of Ireland, Maynooth (Ireland) and Univ. of Oulu (Finland)

707209 Segmentation of three-dimensional scenes encoded in digital holograms [7072-08] K. M. Molony, C. P. McElhinney, B. M. Hennelly, National Univ. of Ireland, Maynooth (Ireland); T. J. Naughton, National Univ. of Ireland, Maynooth (Ireland) and Univ. of Oulu (Finland)

7072 0A Coherence holography and singular optical coherence (Invited Paper) [7072-09] M. Takeda, The Univ. of Electro-Communications (Japan); W. Wang, Heriot-Watt Univ. (United Kingdom)

7072 0C Characterizations of multi-segment DFB laser [7072-11]

S. Mohseni, B. Alhasson, M. A. Matin, Univ. of Denver (United States) 
7072 OD Measuring chromatic dispersion using single-arm interferometers: from millimeters to kilometers (Invited Paper) [7072-12]

L. Qian, B. Qi, W. S. Mohammed, M. A. Galle, F. Ye, Univ. of Toronto (Canada)

7072 OE Simulating paraxial optical systems using the linear canonical transform: properties, issues, and applications [7072-13]

J. J. Healy, P. O'Grady, J. T. Sheridan, Univ. College Dublin (Ireland)

7072 OF Point spread function (PSF) measurement for cell phone camera with a high resolution PSF of the imaging lens and a sub-pixel digital algorithm [7072-14]

C. Liu, X. Chen, Micron Technology, Inc. (United States)

7072 OG A compound-eye imaging system with irregular lens-array arrangement [7072-15]

R. Horisaki, Osaka Univ. (Japan); Y. Nakao, T. Toyoda, Funai Electric Co., Ltd. (Japan);

K. Kagawa, Osaka Univ. (Japan); Y. Masaki, Funai Electric Co., Ltd. (Japan); J. Tanida,

Osaka Univ. (Japan)

$7072 \mathrm{OH} \quad$ Package inspection using inverse diffraction [7072-16]

A. D. McAulay, Lehigh Univ. (United States)

7072 Ol Aperture synthesis imaging through furbulence with the aid of lucky imaging [7072-17] J. E. Ward, Univ. College Dublin (Ireland); W. T. Rhodes, Florida Atlantic Univ. (United States);

J. T. Sheridan, Univ. College Dublin (Ireland)

\section{SESSION 4 COMPUTING}

7072 OL A defect inspection technique using polarized images for steel strip surface [7072-21]

A. Kazama, T. Oshige, JFE R\&D Corp. (Japan)

7072 OM Ultra-fast wave-optical computing [7072-22]

T. Haist, Univ. Stuttgart (Germany)

7072 OP Three-channel dynamic photometric stereo: a new method for 4D surface reconstruction and volume recovery [7072-25]

W. Schroeder, W. Schulze, T. Wetter, Ruprecht-Karls-Univ. Heidelberg (Germany);

C.-H. Chen, Taipei Medical Univ., Wan Fang Hospital (Taiwan) and Taipei Medical Univ. (Taiwan)

$70720 Q \quad$ Temperature and field effects on reflectivity of gallium selenide surface [7072-26]

B. Alhasson, Univ. of Denver (United States); Y. Hajiyev, Technical Univ. of Azerbaijan (Azerbaijan); M. Matin, Univ. of Denver (United States)

7072 OR Which optical processes are suitable to make probabilistic single photon sources for quantum cryptography? [7072-48]

A. Verma, A. Pathak, Jaypee Institute of Information Technology Univ. (India) 
7072 OT Single camera-based object detection and tracking for mobile robots [7072-30]

J. K. Anderson, K. M. Iftekharuddin, E. Threlkeld, B. Montgomery, The Univ. of Memphis (United States)

7072 OU Shadow extraction [7072-31]

K. S. Gudmundsson, Univ. of Iceland (Iceland); A. A. S. Awwal, Lawrence Livermore National Lab. (United States); F. S. Cagatin, Univ. of Iceland (Iceland)

7072 OV Higher accuracy template for corner cube reflected image [7072-32]

A. A. S. Awwal, Lawrence Livermore National Lab. (United States); K. L. Rice, Clemson Univ. (United States); R. R. Leach, Lawrence Livermore National Lab. (United States); T. M. Taha, Clemson Univ. (United States)

7072 oW Two-dimensional pattern processing by means of image compression [7072-33] K. Nitta, T. Minami, O. Matoba, Kobe Univ. (Japan)

7072 OY Enhanced 3D correlator using Blur metric-based resolution enhancement [7072-45] K.-J. Eun, K.-J. Lee, D.-C. Hwang, E.-S. Kim, Kwangwoon Univ. (Korea, Republic of)

\section{POSTER SESSION}

$70720 Z$ Cost function statistical analysis in double random phase encoding [7072-35]

D. S. Monaghan, Univ. College Dublin (Ireland); U. Gopinathan, G. Situ, Univ. Stuttgart (Germany); T. J. Naughton, National Univ. of Ireland, Maynooth (Ireland) and Univ. of Oulu (Finland); J. T. Sheridan, Univ. College Dublin (Ireland)

707210 An alternative design of holographic polarization-selective elements [7072-36] J.-H. Chen, K.-H. Chen, J.-P. Liu, Feng Chia Univ. (Taiwan); J.-Y. Lin, National Changhua Univ. of Education (Taiwan); N.-Y. WU, Feng Chia Univ. (Taiwan)

707211 Complex shadow extraction [7072-37]

K. S. Gudmundsson, F. S. Cagatin, Univ. of Iceland (Iceland)

707213 Multi-beam coupling in doubly-doped photorefractive LiNbO $3: \mathrm{Fe}: \mathrm{Mn}$ crystals [7072-40] C. Dai, Y. Yu, Shanghai Univ. (China); D. Liu, L. Liu, Shanghai Institute of Optics and Fine Mechanics (China)

707214 An improved quantum key distribution protocol [7072-41]

T. Wu, South China Univ. of Technology (China); G. Wu, South China Univ. of Technology (China) and Guangdong Mobile Communication Co., Ltd. (China)

707215 A practical guide to digital holography and generalized sampling [7072-42]

D. P. Kelly, B. M. Hennelly, C. McElhinney, National Univ. of Ireland/Maynooth (Ireland); T. J. Naughton, National Univ. of Ireland/Maynooth (Ireland) and Univ. of Oulu (Finland) 
707216 A method to reduce the size of LUT for generation of a full-color CGH using a relation between distance and wavelength in Fresnel diffraction [7072-43]

J.-H. Yoon, S.-C. Park, S.-C. Kim, E.-S. Kim, Kwangwoon Univ. (Korea, Republic of)

707218 Modeling and experiment of future hearing aid device [7072-46]

H. Tang, Y.-B. Yi, M. A. Matin, Univ. of Denver (United States)

$70721 \mathrm{~A}$ Decoding software of computer instruction codified as Fourier holograms in a LiNbO3:Fe [7072-52]

E. E. Rodríguez Vázquez, E. Tepichín-Rodríguez, Instituto Nacional de Astrofísica, Óptica y Electrónica (Mexico)

7072 1B Digital holographic XNOR multi-gate (DHMG-XNOR) [7072-53]

E. E. Rodriguez Vázquez, E. Tepichin-Rodríguez, Instituto Nacional de Astrofísica, Óptica y Electrónica (Mexico)

7072 1C Phase retrieval in digital holographic microscopy using a Gerchberg-Saxton algorithm [7072-54]

M.-L. Cruz, A. Castro, Instituto Nacional de Astrofísica, Óptica y Electrónica (Mexico); V. Arrizón, Instituto Nacional de Astrofísica, Óptica y Electrónica (Mexico) and Univ. de Sonora (Mexico)

7072 1D Defection of circuit-board components with an adaptive multiclass correlation filter [7072-55]

V. H. Diaz-Ramirez, Instituto Tecnologico de Mexicali (Mexico); V. Kober, CICESE (Mexico)

7072 IE Acousto-optical data processing exploiting the wave heterodyning [7072-56]

A. S. Shcherbakov, National Institute for Astrophysics, Optics, and Electronics (Mexico); O. I. Belokurova, State Polytechnic Univ. (Russia); E. Tepichin Rodriguez, D. Sánchez Lucero, National Institute for Astrophysics, Optics, and Electronics (Mexico)

7072 IF Signal processing for hologram pattern generation in an image system with multi-vision capturing and wavefront reconstruction [7072-57]

K. Nitta, T. Kurahashi, N. Nishikawa, O. Matoba, Kobe Univ. (Japan)

7072 IG Near lossless medical image compression using JPEG-LS and cubic spline interpolation [7072-58]

T.-C. Lin, C.-W. Chen, I-Shou Univ. (Taiwan); S.-H. Chen, Shu-Te Univ. (Taiwan); T.-K. Truong, I-Shou Univ. (Taiwan)

$70721 \mathrm{~K}$ Distortion-invariant target detection using shifted-reference joint transform correlator [7072-65]

M. N. Islam, K. V. Asari, M. A. Karim, Old Dominion Univ. (United States); M. S. Alam, Univ. of South Alabama (United States)

$70721 \mathrm{M}$ A new mean filter ratio technique for edge detection and foreground extraction [7072-67] M. M. Islam, M. N. Islam, K. V. Asari, Old Dominion Univ. (United States); M. S. Alam, Univ. of South Alabama (United States) 
$70721 \mathrm{~N}$ Superresolution image reconstruction using panchromatic and multispectral image fusion [7072-68]

M. I. Elbakary, M. S. Alam, Univ. of South Alabama (United States)

Author Index 
Downloaded From: https://www.spiedigitallibrary.org/conference-proceedings-of-spie on 26 Apr 2023

Terms of Use: https://www.spiedigitallibrary.org/terms-of-use 


\title{
Conference Committee
}

\author{
Program Track Chair
}

Khan M. Iftekharuddin, The University of Memphis (United States)

Conference Chairs

Abdul Ahad Sami Awwal, Lawrence Livermore National Laboratory (United States)

Khan M. Iftekharuddin, The University of Memphis (United States)

Bahram Javidi, University of Connecticut (United States)

Program Committee

Henri H. Arsenault, Université Laval (Canada)

George Barbastathis, Massachusetts Institute of Technology (United States)

Fred Richard Beyette, Jr., University of Cincinnati (United States)

David P. Casasent, Carnegie Mellon University (United States)

H. John Caulfield, Fisk University (United States)

Yeshaiahu Fainman, University of California, San Diego (United States)

Pietro Ferraro, Istituto Nazionale di Ottica Applicata (Italy)

James G. Grote, Air Force Research Laboratory (United States)

Laurence G. Hassebrook, University of Kentucky (United States)

Kazuyoshi Itoh, Osaka University (Japan)

Mohammad A. Karim, Old Dominion University (United States)

Yao Li, Alliance Fiber Optic Products Inc. (United States)

Robert Magnusson, University of Connecticut (United States)

Abhijit Mahalanobis, Lockheed Martin Missiles and Fire Control (United States)

Manuel Martinez-Corral, Universidad de València (Spain)

Mohammad A. Matin, University of Denver (United States)

Osamu Matoba, Kobe University (Japan)

Alastair D. McAulay, Lehigh University (United States)

Maria S. Millan Garcia-Varela, Universitat Politècnica de Catalunya (Spain)

Nasser M. Nasrabadi, Army Research Laboratory (United States)

Thomas J. Naughton, National University of Ireland/Maynooth (Ireland)

Takanori Nomura, Wakayama University (Japan)

Elisabet Pérez-Cabré, Universitat Politècnica de Catalunya (Spain)

Ting-Chung Poon, Virginia Polytechnic Institute and State University (United States)

Philippe Réfrégier, Institut Fresnel (France) 
Nabeel Agha Riza, College of Optics \& Photonics, University of Central Florida (United States)

Joseph Rosen, Ben-Gurion University of the Negev (Israel)

Firooz A. Sadjadi, Lockheed Martin Corporation (United States)

John T. Sheridan, National University of Ireland, Dublin (Ireland)

Jung-Young Son, Hanyang University (South Korea)

Clay James Stanek, DataPath, Inc. (United States)

Enrique Tajahuerce, Universidad Jaume I (Spain)

Jun Tanida, Osaka University (Japan)

Shyh-Lin Tsao, National Taiwan Normal University (Taiwan)

Kelvin H. Wagner, University of Colorado at Boulder (United States)

Cardinal Warde, Massachusetts Institute of Technology (United States)

Frank Wyrowski, Friedrich Schiller Universität Jena (Germany)

Toyohiko Yatagai, University of Tsukuba (Japan)

Francis T. S. Yu, The Pennsylvania State University (United States)

Maria Josefa Yzuel, Universitat Autònoma de Barcelona (Spain)

\section{Session Chairs}

$1 \quad$ Switching

Lawrence R. Chen, McGill University (Canada)

2 Holography/Modeling

Abdul Ahad Sami Awwal, Lawrence Livermore National Laboratory

(United States)

$3 \quad$ Imaging

Mohammad A. Matin, University of Denver (United States)

$4 \quad$ Computing

Khan M. Iftekharuddin, The University of Memphis (United States)

$5 \quad$ Image Processing

Karl S. Gudmundsson, University of Iceland (Iceland) 